\title{
Atypical Presentation of COVID-19 in a Patient with Polysubstance Use and Retroviral Positive Status
}

\author{
Sunil KG Patil ${ }^{1}$, Ume Hani ${ }^{2}$, Khushboo Dewani ${ }^{3}$, Shivakumar Thandavamurthy ${ }^{4}$, Mahesh R Gowda ${ }^{5}$
}

\begin{abstract}
Aim: To know the uncommon presentations of coronavirus disease 2019 (COVID-19) in patients with psychiatric illness for early diagnosis. Background:The COVID-19 pandemic has been associated with multiple mental health issues. A recent survey by the Indian Psychiatric Society also highlighted the presence of stress, anxiety, depression, insomnia, fear, and cognitive defects in patients with COVID-19. The occurrence of COVID-19 in people with the psychiatric illness may present with various challenges right from diagnosis to treatment.

Case description: A 41-year-old male with a history of multiple substance use, in dependence pattern for alcohol, opioids, benzodiazepines, Cannabis, and tobacco, since the past 20 years presented with a history of generalized fatigue for 1 day and drowsiness since morning. The patient was on antiretroviral treatment since 4 years. The patient had a history of multiple attempts of deliberate self-harm (DSH) of impulsive type and a history of an overdose of prescribed medicines (quetiapine and nitrazepam) as well. In view of the history of DSH, drug overdose was suspected in the absence of the history of reuse of substances. However, the real-time polymerase chain reaction (RT-PCR) was positive for severe acute respiratory syndrome coronavirus 2 (SARS-CoV-2), and a minimal improvement happened on drug overdose-related treatment. Conclusion: These patients pose a challenge in the prevention and control of COVID-19 spread. It also hinders the diagnosis and effective management leading to further complications. Among patients with polysubstance use and also among immunocompromised populations, the presence of atypical symptoms should raise a suspicion of COVID-19 and screening protocols should be revaluated.

Clinical significance: A comprehensive understanding of the atypical symptoms should be helpful in identifying and effectively managing COVID-19. This also emphasizes the use of appropriate protection during clinical contact with every patient keeping in mind the risk of infection. Keywords: Atypical presentation, COVID-19, Drug overdose, Pandemic. Indian Journal of Private Psychiatry (2021): 10.5005/jp-journals-10067-0083
\end{abstract}

\section{BACKGROUND}

The coronavirus disease 2019 (COVID-19) pandemic has been associated with multiple mental health issues. A recent survey by the Indian Psychiatric Society also highlighted the presence of stress, anxiety, depression, insomnia, fear, and cognitive defects in patients with COVID-19. ${ }^{1}$ The occurrence of COVID-19 in people with the psychiatric illness may present with various challenges right from diagnosis to treatment. It is of paramount importance to know the uncommon presentations of COVID-19 in patients with psychiatric illness for early diagnosis.

\section{Case Description}

A 41-year-old male presented to casualty with a history of generalized fatigue for 1 day and drowsiness since morning.

The patient had a history of multiple substance use, in dependence pattern for alcohol, opioids, benzodiazepines, Cannabis, and tobacco since the past 20 years. Though he was currently abstinent for opioids for the past 2 years, he had regular use of tobacco smoking and occasional consumption of alcohol, not amounting to dependence.

The patient was diagnosed to be retroviral positive about 4 years ago and was on antiretroviral treatment. The patient had a history of multiple attempts of deliberate self-harm (DSH) of impulsive type and overdose of prescribed medicines as well (quetiapine and nitrazepam).

On examination, the patient was drowsy, not responding to oral commands. His pulse rate was $92 \mathrm{bpm}$, blood pressure was normal, and his saturation recorded at room air was 90 .

\footnotetext{
${ }^{1}$ Department of Psychiatry, Sri Siddhartha Medical College, Tumkur, Karnataka, India

${ }^{2}$ Department of Psychiatry, MVJ Medical College and Research Hospital, Bengaluru, Karnataka, India

${ }^{3}$ Department of Psychiatry, S Nijjalingappa Medical College and HSK Hospital, Bengaluru, Karnataka, India

${ }^{4,5}$ Department of Psychiatry, Spandana Health Care, Bengaluru, Karnataka, India
}

Corresponding Author: Ume Hani, Department of Psychiatry, MVJ Medical College and Research Hospital, Bengaluru, Karnataka, India, Phone: +91 9972959545, e-mail: haani29@yahoo.com

How to cite this article: Patil SKG, Hani U, Dewani K, et al. Atypical Presentation of COVID-19 in a Patient with Polysubstance Use and Retroviral Positive Status. Ind J Priv Psychiatry 2021;15(2):98-100.

Source of support: Nil

Conflict of interest: None

In view of the history of DSH, drug overdose (nitrazepam) was suspected in the absence of the history of reuse of substances. Urine drug screen showed benzodiazepine to be positive, for which a gastric lavage was given and an injection flumazenil $200 \mu \mathrm{g}$ was given stat. The patient had a transient improvement in consciousness but continued to be drowsy later.

On further checking, due to fluctuating saturation levels from 70 to $94 \%$, high-resolution computed tomography (HRCT) of the thorax (Fig. 1) was done, and the COVID-19 Reporting and Data System score was found to be 5 and the CT severity score was 20/25 (severe).

(c) The Author(s). 2021 Open Access This article is distributed under the terms of the Creative Commons Attribution 4.0 International License (https://creativecommons. org/licenses/by-nc/4.0/), which permits unrestricted use, distribution, and non-commercial reproduction in any medium, provided you give appropriate credit to the original author(s) and the source, provide a link to the Creative Commons license, and indicate if changes were made. The Creative Commons Public Domain Dedication waiver (http://creativecommons.org/publicdomain/zero/1.0/) applies to the data made available in this article, unless otherwise stated. 


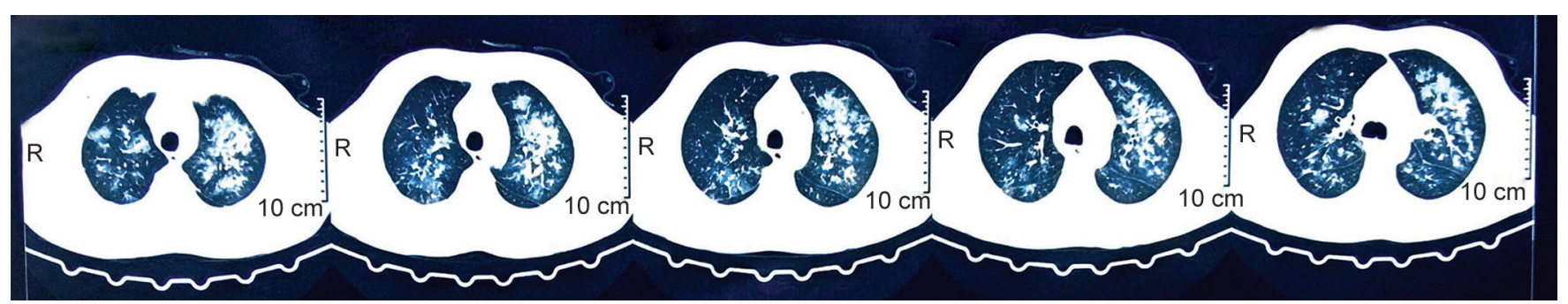

Fig. 1: HRCT thorax

White blood counts were 13,800, D-dimer-2250 ng/mL, and lactate dehydrogenase $-572 \mathrm{U} / \mathrm{L}$, and the following day his real-time polymerase chain reaction (RT-PCR) was positive for SARS-CoV-2.

The patient was shifted to COVID-19 center and was treated for COVID-19. He responded well to the treatment and then recovered. The patient and attenders later clarified the history that he did not reuse substances neither he abused any medications.

\section{Discussion}

COVID-19 is a novel infectious disease with an evolving understanding of its clinical manifestations. A global pandemic of COVID-19 was declared by the World Health Organization on March 11, 2020. ${ }^{2}$

Among reported cases, the most frequent manifestation appears to be pneumonia. Characterized primarily by fever and cough and usually accompanied by dyspnea, on chest imaging found to have bilateral infiltrates. ${ }^{3}$ However, it was found that some patients did not present with these typical manifestations. And about $26.58 \%$ of COVID-19 patients had an atypical presentation. ${ }^{4}$ Among the found atypical symptoms, diarrhea, confusion without fever, or cough was found to be common. ${ }^{5}$

The exact pathophysiology behind the atypical symptoms is not clearly understood, but some factors contributing and increasing the probability of these symptoms might be advanced age and many comorbidities. ${ }^{6}$ In immunocompromised, the patient having COVID-19 may sometimes present with atypical clinical manifestations. ${ }^{7}$ In patients with chronic immunosuppression, atypical presentation of respiratory infections may be encountered, often with simultaneous two or more infectious processes. ${ }^{8}$ Patients with substance use disorders (smoking, opioid users, and Cannabis users), both past and current, are at increased risk of COVID-19. ${ }^{9}$ Chronic respiratory distress is found to be common among opioid users. ${ }^{10}$ Even mild symptoms or atypical symptoms of COVID-19 might constitute a threat in patients who have been diagnosed with substance use disorder at some point in their lives. ${ }^{11}$

Some clinical and demographic characteristics have been associated with the severity of COVID-19, such as immunosuppression, chronic respiratory disease, diabetes, etc.- -which also might enhance the lethality risk for COVID-19. It would also mean that patients with moderate to severe substance use disorders, who already are an important risk group, could present and suffer with major impacts, since they have been associated with all these conditions previously. ${ }^{12}$ This situation might pose a hindrance in the management of COVID-19, if not diagnosed earlier due to atypical presentation.

We would like to emphasize the challenging scenario faced during the diagnosis of this case. The history of polysubstance use and retroviral positive status of the patient may have been contributing factors for atypical presentation. People with the retroviral disease with low CD4 counts are at an increased risk of COVID infection. Our patient had CD4 counts ranging between 500 and 800 cells $/ \mathrm{mm}^{3}$ in last 3 months. Also, screening CT scan should be advised as it is demonstrated that the sensitivity of the first PCR test has been to be less compared to the chest computed tomography (CT) scan, ${ }^{13}$ and detection of typical chest CT findings of COVID-19 should prompt toward further isolation even if the presentation of chief complaints is atypical.

\section{Conclusion}

These patients pose a challenge in the prevention and control of COVID-19 spread and also hinder the diagnosis and effective management leading to further complications. Clinical suspicion and CT imaging were of significant help in this case for early diagnosis of COVID-19. Nevertheless, among patients with polysubstance use and also among immunocompromised populations, the presence of atypical symptoms should raise a suspicion of COVID-19 and screening protocols should be revaluated.

\section{Clinical Significance}

A comprehensive understanding of the atypical symptoms should be helpful in identifying and effectively managing COVID-19. This also emphasizes the use of appropriate protection during clinical contact with every patient keeping in mind the risk of infection.

\section{References}

1. Grover S, Sahoo S, Mehra A, et al. Psychological impact of COVID-19 lockdown: An online survey from India. Indian J Psychiatry 2020;62(4):354. DOI: 10.4103/psychiatry.IndianJPsychiatry_427_20.

2. Coronavirus Disease (COVID-19) - events as they happen 2020. Available from: https://www.who.int/emergencies/diseases/novelcoronavirus-2019/events-as-they-happen.

3. Huang C, Wang Y, Li X, et al. Clinical features of patients infected with 2019 novel coronavirus in Wuhan, China. Lancet 2020;395(10223): 497-506. DOI: 10.1016/S0140-6736(20)30183-5.

4. Yang Z, Chen X, Huang, R, et al. Atypical presentations of coronavirus disease 2019 (COVID-19) from onset to readmission. BMC Infect Dis 2021;21:127. DOI: 10.1186/s12879-020-05751-8.

5. Tin SS, Wiwanitkit V. Uncommon atypical presentations of COVID-19: important and should not be under recognized! J Health Sci Med Res 2020;38(2):153-158. DOI: 10.31584/jhsmr.2020733.

6. Atypical symptoms in COVID-19: the many guises of a common culprit: 2020. Available from: http://www.bmj.com/content/369/bmj m1375/rr-12.

7. Guillen E, Pineiro GJ, Revuelta I, et al. Case report of COVID-19 in a kidney transplant recipient: does immunosuppression alter the clinical presentation? Am J Transplant 2020;20(7):1875-1878. DOI: 10.1111/ ajt.15874. 
8. Shelhamer JH, Toews GB, Masur $\mathrm{H}$, et al. Respiratory disease in the immunosuppressed patient. Ann Intern Med 1992;117(5):415-431. DOI: 10.7326/0003-4819-117-5-415.

9. National Institute on Drug Abuse. COVID19: potential implications for individuals with substance use disorders; 2020. Available from: https://www.drugabuse.gov/aboutnida/norasblog/2020/03/ covid19potentialimpicationsindividualssubstanceuse disorders.

10. Hulin J, Brodie A, Stevens J, et al. Prevalence of respiratory conditions among people who use illicit opioids: a systematic review. Addiction (Abingdon, England) 2019;115(5):832-849. DOI: 10.1111/add.14870.

11. National Institute on Drug Abuse. Substance use disorders linked to COVID-19 susceptibility: 2020. Available from: https://www.nih. gov/news-events/newsreleases/substance-use-disorders-linkedcovid-19-susceptibility.

12. Lagisetty PA, Maust $D$, Heisler $M$, et al. Physical and mental health comorbidities associated with primary care visits for substance use disorders. J Addict Med 2017;11(2):161-162. DOI: 10.1097/ ADM.0000000000000280.

13. Mirahmadizadeh A, Pourmontaseri Z, Afrashteh S, et al. Sensitivity and specificity of chest computed tomography scan based on RT-PCR in COVID-19 diagnosis. Pol J Radiol 2021;86:e74-e77. DOI: 10.5114/pjr.2021.103858. 